\title{
Multiplication par bouturage de Jatropha curcas $L$. : influence du nombre de nœuds, du diamètre, de la durée et du temps de conservation des boutures
}

\author{
Amsatou THIAM ${ }^{1 *}$, Samba Arona Ndiaye SAMBA ${ }^{2}$, Cheick Omar SAMB ${ }^{3}$ et \\ Ablaye NIANG ${ }^{1}$
}
${ }^{1}$ Université Alioune Diop Bambey, Institut Supérieur de Formation Agricole et Rurale (ISFAR), BP 54, Bambey, Sénégal.
${ }^{2}$ Université de Thiès Ecole Nationale Supérieure d'Agriculture (ENSA), BP 967, Thiès, Sénégal.
${ }^{3}$ Institut Sénégalais de Recherches Agricoles/Centre National de recherches Forestières (ISRA/CNRF).
*Auteur correspondant ; E-mail: amsathiam@yahoo.fr

\section{RESUME}

Depuis l'avènement des chocs pétroliers, beaucoup de pays se sont interrogés sur le problème de l'énergie. Ainsi, de nombreuses études ont été menées dans le but de résorber ce problème énergétique qui touche en grande partie les pays africains tel que le Sénégal. Ces dernières ont porté sur l'utilisation de sources d'énergies renouvelables comme le Jatropha curcas L. qui présente de nombreux avantages biophysiques et médicaux. La présente étude est réalisée dans l'objectif d'expérimenter l'effet de la conservation dans le temps de différentes gammes de boutures sur son débourrement. Différentes catégories de boutures (boutures à 6 nœuds et à 4 nœuds) ayant chacune proportionnellement des classes de diamètres comprises entre]0-1] et] 1-2] $(\mathrm{cm})$ ont été utilisées. Elles ont été conservées en chambre froide et à l'air libre sur une durée de 45 jours. Chaque $15^{\text {ème }}$ jour un lot de même proportion de boutures conservées dans les deux conditions est planté en partant des boutures témoins plantées juste après récolte. Avant plantation, le statut hydrique des différentes catégories de boutures sont relevées à bases d'indicateurs tels que le potentiel hydrique et la teneur en eau. L'évaluation de l'état hydrique des boutures avec la teneur en eau a donné $(86,93 \%)$ pour les boutures conservées en chambre froide, et (67,31\%) pour celles conservées à l'air libre. Cet effet est réversible avec le potentiel hydrique qui donne respectivement $(-0,33 \%)$ et $(-0,69 \%)$. Le débourrement est maximal avec le témoin pour les deux modes de conservation et régresse en fonction du temps de conservation (témoin, 15 jours, 30 jours, 45 jours). Malgré cet effet, les boutures conservées à l'air libre ont connu un meilleur débourrement $(92,5 \%, 70,83 \%, 67,5 \%, 42,08 \%)$ que celles conservées en chambre froide $(85 \%, 32,5 \%, 25 \%$, $0 \%$ ). Cette régression est ainsi assimilée à une augmentation de sa durée de débourrement dans le temps. La teneur en eau, ainsi que le potentiel hydrique varient en fonction du calibre, du mode et du temps de conservation. Cependant, le débourrement des boutures est influencé à la fois par le calibre, le mode et le temps de conservation des boutures

(C) 2019 International Formulae Group. All rights reserved

Mots clés: Jatropha curcas L., boutures, potentiel, teneur, débourrement, conservation. 


\title{
Propagation by cuttings from Jatropha curcas L.: number of nodes effect, diameter, duration and time storage of the cuttings
}

\begin{abstract}
Since the advent of the oil crises, many countries have wondered about the problem of energy.Thus, many studies were carried out in order to find a way of resolving this energy issue which mainly touches the African countries such as Senegal. The latter are related to the use of renewable energies sources such as Jatropha curcas L. which has many biophysical and medical advantages. The present study is carried out with the purpose of trying out the effect of the storage within given time of various ranges of cuttings at its bud break. Various categories of cuttings (cuttings with 6 nodes and 4 nodes) having each one, proportionally, classes of diameters included between] 0-1] and] 1-2] (cm) were used. They were stored in cold room and into the open air during 45 days. Each $15^{\text {th }}$ day, a batch of the same proportion of cuttings stored under the two conditions are planted on the basis of the experimental cuttings planted just after harvest. Before plantation, the water status of the various categories of cuttings are recorded as indicators such as the water potential and the water content. The water status evaluation of the cuttings with the water content has given $86,93 \%$ for the cuttings preserved in cold room, and $67,31 \%$ for those stored into the open air. This effect is reversible with the water potential which gives respectively $(-0,33 \%)$ and $(-0,69 \%)$. The bud break is at the highest level with the experimental cuttings for the two ways of storage and regresses according to the time of storage (experimental cutting, 15 days, 30 days, and 45 days). Despite this effect, the cuttings stored into the open air knew the best bud break $(92.5 \%, 70.83 \%, 67.5 \%$, and $42.08 \%)$ that those preserved in cold room $(85 \%, 32.5 \%, 25 \%$, and $0 \%)$. This regression is thus related to an increase of the bud break duration over time. The water content, as well as the water potential varies according to the cuttings diameter, the way and the duration of their storage. However, the bud break of the cuttings is influenced at the same time by the diameter, the way and the duration of the cuttings storage.
\end{abstract}

(C) 2019 International Formulae Group. All rights reserved

Keywords: Jatropha curcas L., cuttings, potential, content, bud break, storage

\section{INTRODUCTION}

Le carburant est au centre de l'activité économique mondiale et fournit l'énergie essentielle pour la production des biens matériels, indispensables pour le développement d'un pays. Cette source d'énergie qui se présente en majeure partie sous forme fossile (pétrole, gaz, houille), avec une représentativité de $80 \%$ au niveau mondial (Ecomet, 2014), pèse lourdement sur le produit intérieur brut (PIB) des Pays Africains surtout ceux Non Producteurs de Pétrole (PANPP). Parmi ces pays, le Sénégal n'est pas en reste et dépend à plus de $44,6 \%$ d'approvisionnements extérieurs en énergie fossile pour satisfaire sa consommation en énergie (SIE-Sénégal, 2010).

Face à ces problèmes, le recours aux bioénergies en général et aux biocarburants en particulier constitue une alternative capitale. Au Sénégal Ricinus communis L (ricin) figure parmi les espèces du Programme Biocarburant au Sénégal. Sa culture sur des terrains incultes (terres salées) constitue une alternative pour éviter une concurrence sur les terres à vocation agricole (Samba et al., 2013). C'est ainsi que de nombreux programmes ont porté un choix sur Jatropha curcas L. du fait de sa qualité, contrairement aux autres types d'agrocarburants issus des plantes oléagineuses tels que le tournesol, le soja, le colza, le ricin et le coton, qui nécessitent impérativement un mélange avec le diesel conventionnel jusqu'à une proportion de 30\% (PLAN REVA)). Au Burkina le développement de Jatropha est surtout l'œuvre des promoteurs privés ou de projets comme GENESE SARL, APROJER et AGRITECH FASO (Bazongo et al., 2015).

En effet, au-delà de ses potentialités pour la production de biocarburant, Jatropha curcas $L$. présente de nombreux autres avantages. Il a la capacité de s'adapter aux conditions semi-arides et aux sols pauvres et de se propager facilement par semis ou par bouturage (Achten et al., 2008, cité par Barbier, 2012). L'induction artificielle permet d'envisager de nouvelles perspectives de multiplication (voire de domestication) de 
certaines espèces agroforestières très demandées. (Arnaud Wendpouire et al., 2014). De plus, Jatropha curcas L. peut fournir divers produits et sous-produits qui contribuent aux principaux objectifs du développement rural (Openshaw, 2000; Henning, 2001) : la promotion d'activités féminines (production de savon), la réduction de la pauvreté, le maintien de la fertilité des sols par le contrôle de l'érosion.

Cependant, malgré son importance, la culture de Jatropha curcas L. reste confrontée à un problème de diffusion à large échelle. A ce titre, la production massale d'un matériel végétal de plantation de qualité, phénotypiquement homogène devient une préoccupation primordiale. La multiplication végétative (bouturage) est la meilleure alternative pour la production rapide de fruits alors que la propagation générative (semis direct) est intéressant pour une production élevée de biomasse (Nguema Ndoutoumou et al., 2013).

C'est dans cette optique que s'inscrit cette étude dont l'objectif est d'améliorer nos connaissances par rapport aux réponses des boutures du Jatrophas curcas L. en fonction de la durée et des modes de conservations

\section{MATERIEL ET METHODE \\ Matériel \\ Milieu d'étude}

Le dispositif expérimental a été implanté au niveau de la pépinière des Eaux et Forêts située dans la région de Thiès (Sénégal). La région de Thiès est limitée au Nord par la région de Louga, au Sud par la région de Fatick, à l'Ouest par l'océan Atlantique et la région de Dakar et à l'Est par la région de Diourbel. Cette région fait partie du bassin sédimentaire sénégalo-mauritanien. Divers types de sols sont rencontrés dans cette région: des sols à minéraux bruts; des sols peu évolués d'apport (sols peu hydromorphes sur collusions sablo-argileuses), des sols ferrugineux tropicaux faiblement lessivés et des vertisols litho morphes à surface massive modaux sur marne par endroits. Le climat est de type sahélien au Nord et Nord-est, Soudano-sahélien au Sud et Sud-est et de type Sub - canarien à l'Ouest. Les températures varient entre $20,3{ }^{\circ} \mathrm{C}$ et $35{ }^{\circ} \mathrm{C}$ avec une moyenne qui tourne autour de $26{ }^{\circ} \mathrm{C}$. $\mathrm{La}$ région de Thiès enregistre des précipitations dont la moyenne annuelle tourne autour de 300 à $500 \mathrm{~mm}$.

\section{Le Matériel végétal}

Collecte et calibrage des boutures

Les boutures ont été collectées à l'Ecole Nationale Supérieure d'Agriculture de Thiès (ENSA) dans la région de Thiès, sur des pieds intacts n'ayant connu aucun signe de maladie ou d'anomalies par rapport à leur aspect morphologique externe. Le calibrage a consisté à sélectionner des boutures à 3 nœuds et à 6 nœuds appartenant à des classes de diamètre comprises entre] 0 - 1] cm et] 1 - 2] $\mathrm{cm}$.

\section{Méthode \\ Le dispositif expérimental}

Le dispositif expérimental utilisé est un plan en tiroirs (Split x Split x Split plot) à 4 facteurs et 32 traitements répétés 3 fois d'où un nombre total d'unités expérimentales de 96.

Le mode de conservation des boutures constitue le facteur principal à 2 niveaux : (air libre avec une température moyenne de $27{ }^{\circ} \mathrm{C}$; chambre froide $3{ }^{\circ} \mathrm{C}$ ). Le deuxième facteur est le temps de conservation à 4 niveaux (Témoin; 15 jours; 30 jours et 45 jours. Comme troisième facteur on a les différentes classes de diamètre relevées à la base des boutures subdivisées en 2 niveaux:] $0-1$ $\mathrm{cm}] ;] 1-2 \mathrm{~cm}$ ] et enfin on a comme quatrième le nombre de nœuds à deux niveaux : 3 nœuds et 6 nœuds.

(Figure 1. Schéma du dispositif expérimental. Annexe)

\section{Les paramètres observés}

\section{La teneur en eau des boutures}

La teneur en eau a été déterminée selon la méthode gravimétrique. Le poids frais de l'échantillon a été déterminé à partir de pesées avec une balance de précision et le poids sec est obtenu après dessiccation de l'échantillon dans une étude à $105{ }^{\circ} \mathrm{C}$. Cette teneur en eau (TE) s'exprime d'après la relation de Willan (1992): $\mathrm{TE}=\left[\left(\mathrm{P}_{1}-\mathrm{P}_{2}\right) / \mathrm{P}_{1}\right] * 100$ avec $\mathrm{P} 1=$ poids frais et $\mathrm{P} 2$ poids après dessiccation.

\section{Le Potentiel hydrique}

Il est l'indicateur le plus employé pour le diagnostic du stress hydrique de la plante. 
Le calcul du potentiel hydrique s'est effectué en posant sur la presse des portions de boutures de dimensions égales à celles utilisées pour le calcul de la teneur en eau. Des mouvements d'oscillations ont ensuite été effectués sur un levier jusqu'à ce que l'on commence à observer des gouttelettes d'eau sortir des parois de l'échantillon à mesurer. A ce moment on arrête et on relève la donnée observée sur le nanomètre à pression. Celle-ci est donnée en PSI et sera ultérieurement convertie en Mpa (méga pascal) avant traitement des donnés suivant la formule : 1 Psi (pound per square inch) $=0,0068 \mathrm{Mpa}$.

Le taux de reprise

Il désigne la capacité de débourrement des boutures. Il est illustré par le rapport entre le nombre de nœuds ayant débourré sur le nombre total de nœuds pour chaque catégorie de bouture.

TR

= (Nombre de noeuds débourrés|Nombr total de noeuds) * 100

TR : Taux de reprise.

La vitesse de débourrement

Elle est déterminée par la cinétique de débourrement c'est à dire l'évolution du débourrement des catégories de boutures face aux différents traitements.

La durée de reprise des boutures.

C'est le temps qui s'écoule entre le début et la fin de la reprise des boutures pendant la période d'observation.

\section{Analyses statistiques}

Il s'agit d'analyser les interactions entre facteurs et variables étudiées: analyse de la variance et test de corrélation entre les variables.

\section{RESULTATS}

\section{Analyse de variance}

L'analyse de variance au seuil de $5 \%$ que révèle le Tableau 1 , montre que le mode de conservation a une influence très hautement significative sur la teneur en eau $(\mathrm{p}<0,001)$, le potentiel hydrique $(\mathrm{p}<0,001)$, et le taux de débourrement des boutures du Jatropha curcas L. $(\mathrm{p}<0,001)$. Par contre le temps de conservation et le nombre de nœuds ont une influence très hautement significative seulement sur le taux de débourrement $(\mathrm{p}<0,001)$. Par ailleurs, le diamètre des boutures a une influence significative sur la teneur en eau et le potentiel hydrique. (Tableau 1).

\section{Corrélation entre les variables}

Un test de corrélation entre les variables étudiées a montré que la teneur en eau et le potentiel hydrique des boutures sont fortement corrélés $(\mathrm{r}=-0,7992 ; \mathrm{p}<0,001)$. (Tableau 2).

\section{Les Types de conservation sur les boutures en fonction de la durée du stockage Indicateurs hydriques}

Influence du mode et de la durée de stockage sur le potentiel hydrique

L'analyse de la Figure 1 montre une différence significative du potentiel hydrique eu égard aux deux modes de conservation pour toutes les catégories de boutures confondues à un seuil inférieur à 0.001 . Les boutures conservées à l'air libre ont une valeur plus faible avec une moyenne de $(-0,69 \%)$. Cependant, celles conservées en chambre froide sont plus élevées avec en moyenne $(-0,33 \%)$.

Il ressort de l'analyse de la Figure 2 que le potentiel hydrique pour toutes les catégories de boutures confondues varie d'un moment à un autre par rapport à chaque mode de conservation. Le potentiel hydrique des boutures diminue à partir du témoin pour le mode de conservation à l'air libre avec respectivement (-0.39 Mpa ; -0,62 Mpa ; $-0,78$ Mpa ; -1,02 Mpa). Pour les boutures conservées en chambre froide le potentiel hydrique augmente avec le temps de conservation.

Effet du mode et du temps de conservation sur la teneur en eau des boutures

Le mode de conservation des boutures a un effet significatif $(P<0,001)$ sur la teneur en eau. L'observation de la Figure 3 montre que les boutures conservées en chambre froide ont une teneur en eau plus élevée (87\%) que les boutures conservées à l'air libre $(67 \%)$.

L'analyse de la Figure 4 met en évidence que la teneur en eau des boutures conservées en chambre froide augmente en fonction $\mathrm{du}$ temps de conservation contrairement à celles conservées à l'air libre 
Relation entre les deux indicateurs

Sur les Figures 5 et 6 nous constatons que la teneur en eau et le potentiel hydrique sont inversement proportionnels. En effet quand la teneur en eau augmente, le potentiel hydrique diminue. Ces variations du statut hydrique sont visibles eu égard aux modes de conservation mais également au niveau des diamètres des boutures.

\section{Le débourrement}

Analyse du taux de débourrement des boutures de Jatropha curcas L. en fonction du mode et du temps de conservation.

Il ressort de l'analyse de la Figure 7 que le débourrement des différentes catégories de boutures par rapport à leur mode de conservation, a commencé 3 jours après plantation pour les boutures conservées à l'air libre et 5 jours après plantation pour les boutures conservées en chambre froide. Après cette phase, elles suivent une évolution jusqu'au $19^{\mathrm{e}}$ jour avec un taux de $78 \%$ pour les boutures conservées à l'air libre. Cependant pour les boutures conservées en chambre froide le maximum de débourrement est enregistré aux 16 jours pour un taux de $33 \%$. Au-delà ces deux dates, le taux de débourrement demeure constant et ne varie plus jusqu'à la fin du test (43 jours).

L'observation de la Figure 8 montre que le débourrement a commencé 3 jours après plantation sauf pour les boutures conservées pendant 30 jours qui débourrent un peu plus tôt. Après cette phase, une évolution exponentielle de $80 \%$ a été constatée chez les boutures témoins sur une période de 2 jours. Cependant, il est important de noter que le temps de conservation inhibe le débourrement des boutures ce qui fait qu'on observe qu'au fur et à mesure que le temps de conservation s'allonge il y a un ralentissement de la vitesse de débourrement. Ce ralentissement est assimilé à une augmentation de la durée de débourrement des boutures qui atteint respectivement un taux cumulé constant de (12, 16 et 32 jours).

Effet du mode et du temps de conservation sur la cinétique de débourrement des boutures de Jatropha curcas

Il ressort de l'analyse de la Figure 9 que le taux de débourrement varie d'un mode de conservation à un autre pour toutes les catégories de boutures confondues ( $\mathrm{P}<$ 0.001). L'évolution de la capacité de débourrement des boutures en fonction du temps, montre une diminution du taux pour les deux modes de conservation. Mais malgré leur décroissance, les boutures conservées à l'air libre ont montré un meilleur taux de débourrement avec $92,6 \%$ pour le témoin et $42,08 \%$ pour les boutures conservées 45 jours après récolte. Cette régression devient plus accrue pour les boutures conservées en chambre froide avec $(85 \%)$ pour le témoin, qui a tendance à s'annuler au moment de la dernière série de plantation à savoir au $45^{\mathrm{e}}$ jour avec un taux de débourrement de $(0 \%)$.

\section{La reprise}

Analyse du mode de conservation et du temps de conservation sur la durée de reprise des boutures.

La Figure 10 montre la durée de reprise des boutures en fonction du mode de conservation. Les boutures conservées à l'air libre ont enregistré une durée de débourrement moyenne de (3 jours), plus faible chez les boutures conservées en chambre froide qui enregistrent une moyenne de ( 2 jours).

Les résultats de l'analyse (Figure 11) n'ont pas montré un effet significatif du temps de conservation sur la durée de reprise des boutures. Il est en moyenne de 2 à 3 jours.

\section{Les tailles des boutures}

\section{Les indicateurs hydriques}

Influence de la taille des boutures sur le potentiel hydrique et la teneur en eau.

La Figure 12 indique que le classe de diamètre des différentes catégories de boutures a un effet significatif sur le potentiel hydrique $(\mathrm{p}=0,04)$. Les boutures de diamètre compris entre]0-1 cm] ont un potentiel hydrique plus élevé que les boutures de diamètre compris entre] $1-2 \mathrm{~cm}]$.

L'analyse de la Figure 13 montre que le calibre des boutures a une influence sur leur teneur en eau. Ainsi, les boutures à 3 nœuds ont une teneur en eau plus élevée que les boutures à 6 nœuds pour toutes les classes de diamètres confondues. Par ailleurs les boutures ayant un diamètre compris entre]0-1] $\mathrm{cm}$ ont une teneur en eau plus élevée que les boutures de diamètres compris entre]1-2] $\mathrm{cm}$. 


\section{Le débourrement}

Effet du diamètre des boutures et du nombre de nœuds sur la cinétique de débourrement.

L'analyse de la Figure 14 révèle que la cinétique du taux de débourrement des boutures par rapport aux différentes classes de diamètre montre une sensible proportionnalité par rapport à leur évolution. Cependant cette dernière est subdivisée en trois phases :

-une première phase de trois 3 jours appelée temps de latence caractérisé par l'absence de débourrement des boutures ;

-une deuxième phase marquée par un débourrement exponentiel de 11 jours ;

-une troisième phase à partir du $14^{\mathrm{e}}$ jour de débourrement où le taux de germination devient constant pour le restant de l'expérience.

Les courbes observées sur la Figure 15 montrent que la cinétique de débourrement varie selon les deux types de boutures. Les courbes sont caractérisées par la présence de trois phases. Une phase de latence qui dure 3 jours au cours desquels aucun débourrement n'est observé. Une phase exponentielle de 13 jours caractérisée par un taux de débourrement très important et enfin une phase plateau durant laquelle le débourrement est très faible voire nul.

Influence du nombre de nœuds et du calibre des boutures sur le taux de débourrement.

La Figure 16 met en évidence que nombre de nœuds à un effet hautement significatif $(\mathrm{P}<0.001)$ sur le taux de débourrement des boutures de Jatropha curcas L. En effet les boutures à 3 nœuds ont des taux moyens de débourrement plus importants $(69,79 \%$ et $60,41 \%)$ que les boutures à 6 nœuds $(36,25 \%$ et $41,25 \%)$ eu égard à chaque classe de diamètre.

\section{La reprise}

Effet du calibrage sur la durée de reprise des boutures

La Figure 17 montre que le calibre des boutures n'a pas d'effet significatif sur la durée de reprise. Les boutures à 6 nœuds de diamètre] 1-2] $\mathrm{cm}$ ont la durée la plus longue avec une moyenne de $(3,2 \%)$.

Tableau 1 : Analyse de la variance (ANOVA).

\begin{tabular}{lllll}
\hline $\begin{array}{l}\text { Variables } \\
\text { Facteurs }\end{array}$ & $\begin{array}{l}\text { Teneur en } \\
\text { eau }\end{array}$ & $\begin{array}{l}\text { Potentiel } \\
\text { hydrique }\end{array}$ & $\begin{array}{l}\text { Taux de } \\
\text { reprise }\end{array}$ & $\begin{array}{l}\text { Durée de } \\
\text { reprise }\end{array}$ \\
\hline $\begin{array}{l}\text { Mode de conservation } \\
\text { Temps }\end{array}$ & 0.0001 & 0.0001 & 0.0009 & \\
$\begin{array}{l}\text { conservation } \\
\text { Nombre de nœuds }\end{array}$ & & & 0.0001 & \\
Classes de diamètres & 0.0368 & 0.0479 & 0.0000 & \\
\hline
\end{tabular}

Tableau 1 : Corrélation de Pearson.

\begin{tabular}{llllll}
\hline Variables & $\begin{array}{l}\text { Classes } \\
\text { diamètre }\end{array}$ & $\begin{array}{l}\text { Mode } \\
\text { conservation }\end{array}$ & $\begin{array}{l}\text { Nombre } \\
\text { nœuds }\end{array}$ & $\begin{array}{l}\text { Potentiel } \\
\text { hydrique }\end{array}$ & $\begin{array}{l}\text { Taux } \\
\text { débourrement }\end{array}$ \\
\hline mode de conservation & 0,0316 & & & & \\
nombre de nœuds & 0,2121 & $-0,0316$ & & & \\
potentiel hydrique & 0,2692 & $-0,4229$ & 0,1112 & & \\
taux de débourrement & $-0,2261$ & $-0,2177$ & $-0,5521$ & $-0,4206$ & \\
durée de reprise & 0,2267 & $-0,1113$ & 0,181 & 0,1057 & $-0,1049$ \\
teneur en eau & $-0,3407$ & 0,5607 & $-0,3104$ & $\mathbf{- 0 , 7 9 9 2}$ & 0,1362 \\
Temps de conservation & $-0,0516$ & $-0,4257$ & 0,0516 & 0,8656 & $-0,4422$ \\
\hline
\end{tabular}




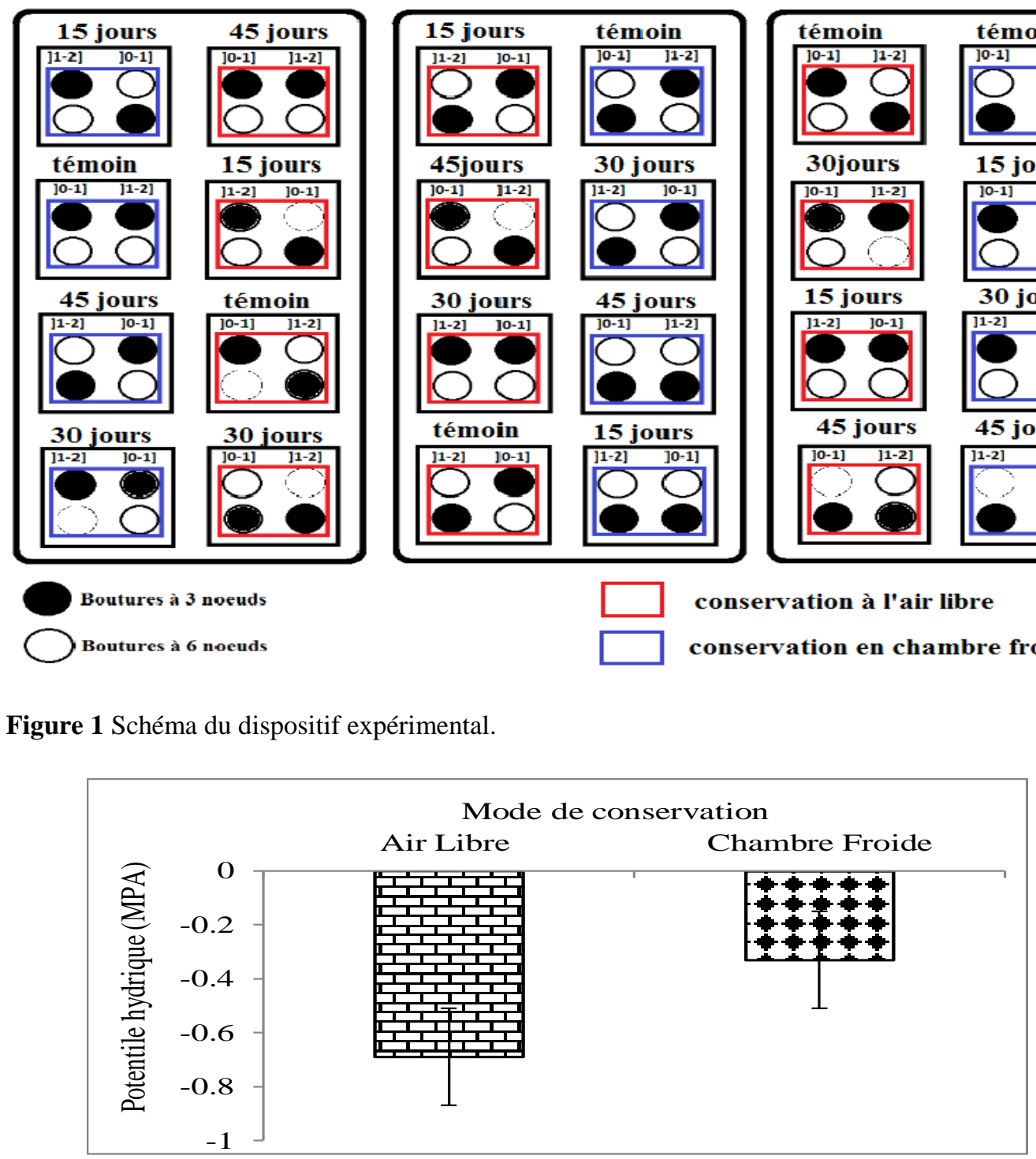

Figure 1. Effet du mode de conservation sur le potentiel hydrique des boutures

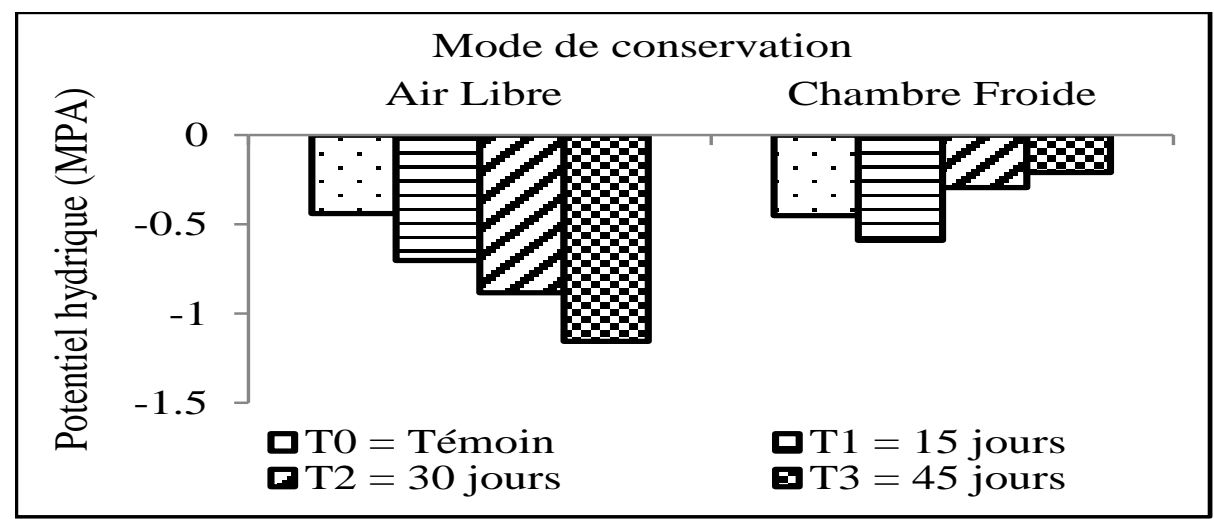

Figure 2. Evolution du potentiel hydrique en fonction du mode et du temps de conservation 


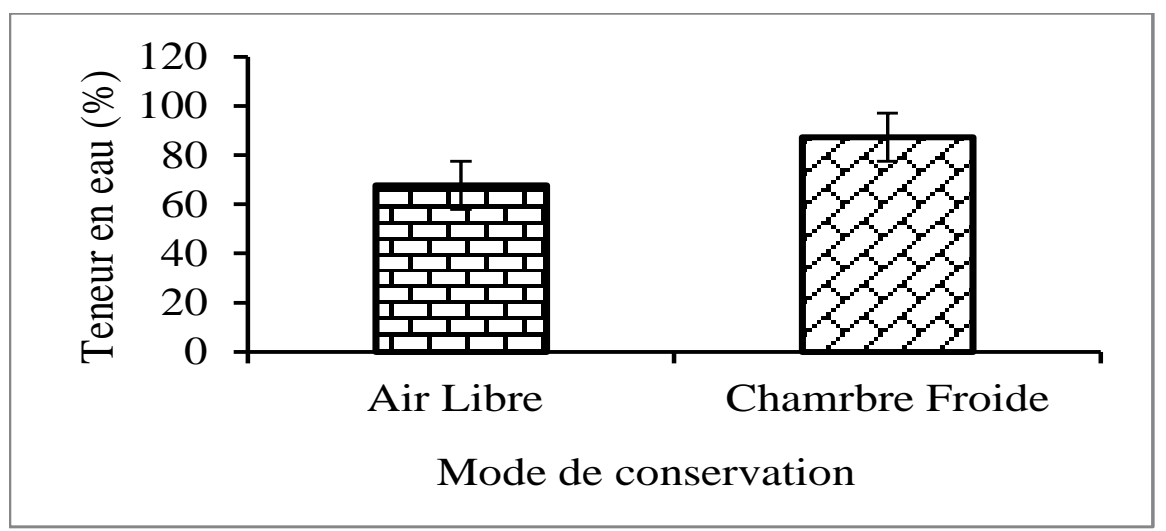

Figure 3. Effet du mode de conservation sur la teneur en eau

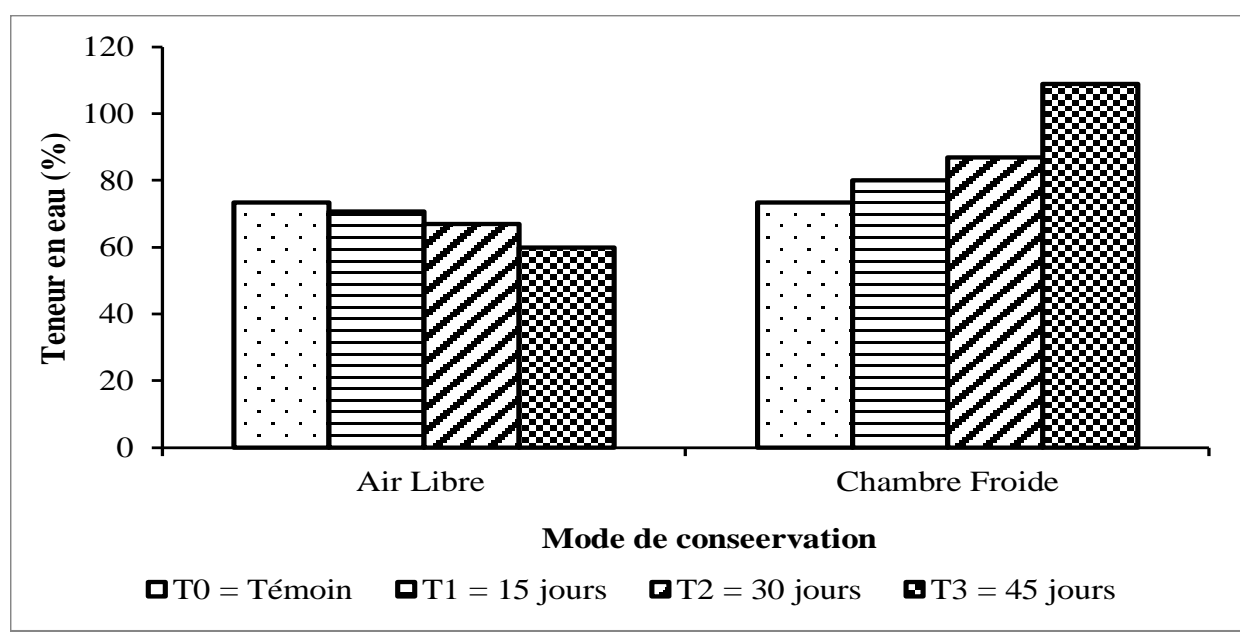

Figure 4. Evolution de la teneur en eau en fonction du mode et du temps de conservation.

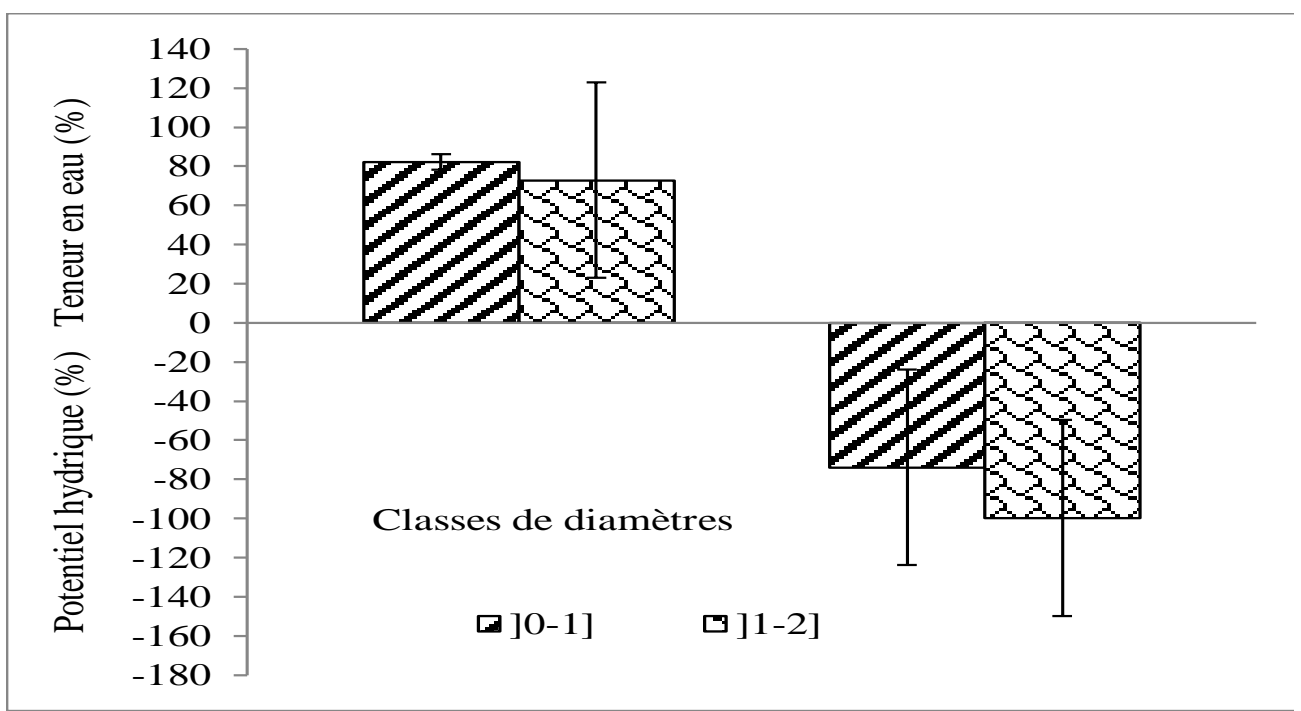

Figure 5. Proportionnalité entre la teneur en eau et le potentiel hydrique des boutures en fonction des classes de diamètre 


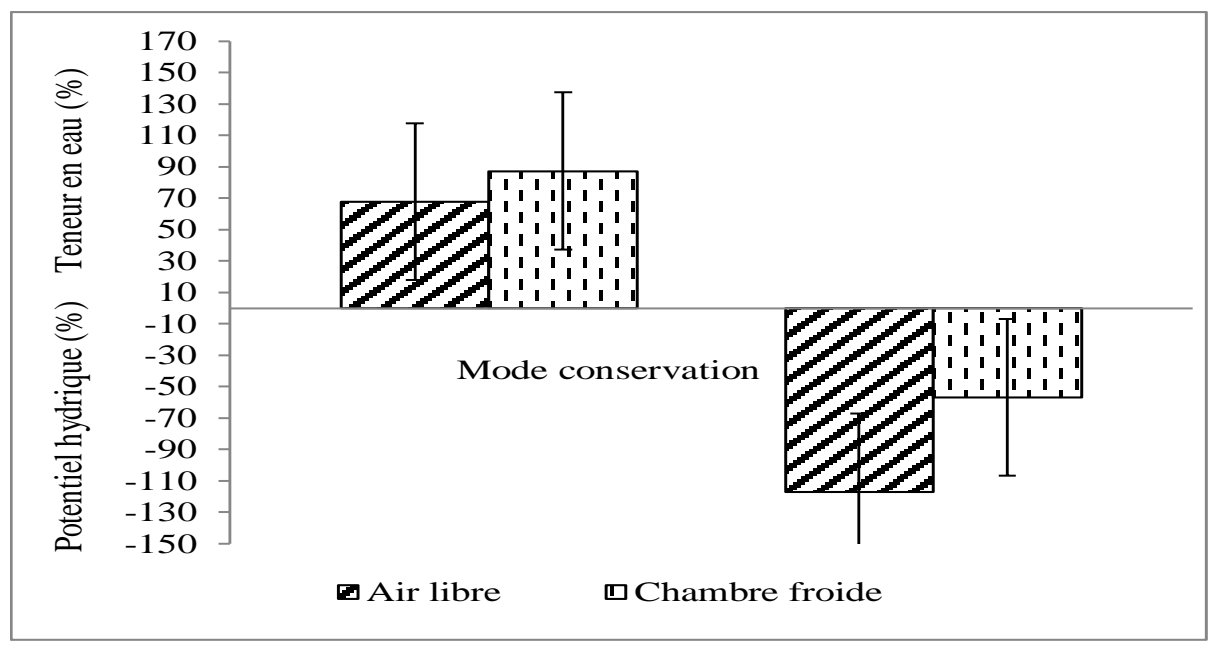

Figure 6. Proportionnalité entre la teneur en eau et le potentiel hydrique des boutures en fonction en fonction des modes de conservation

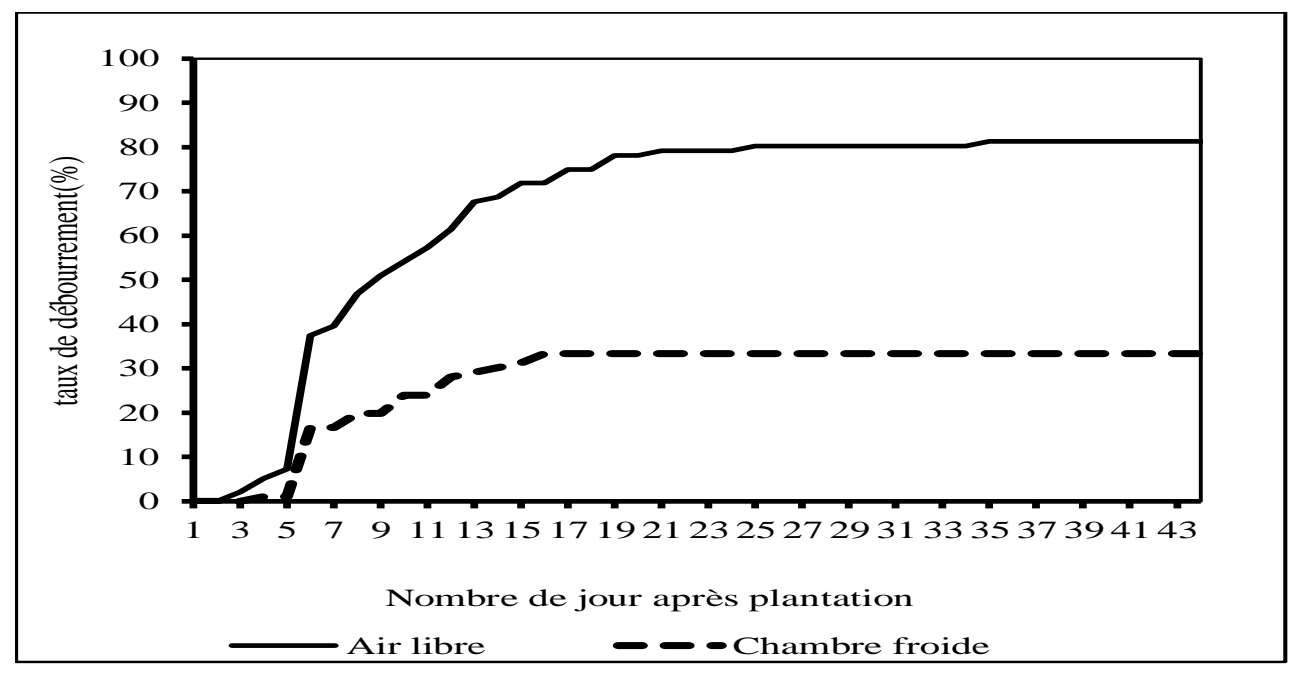

Figure 7. Cinétique de débourrement en fonction du mode de conservation.

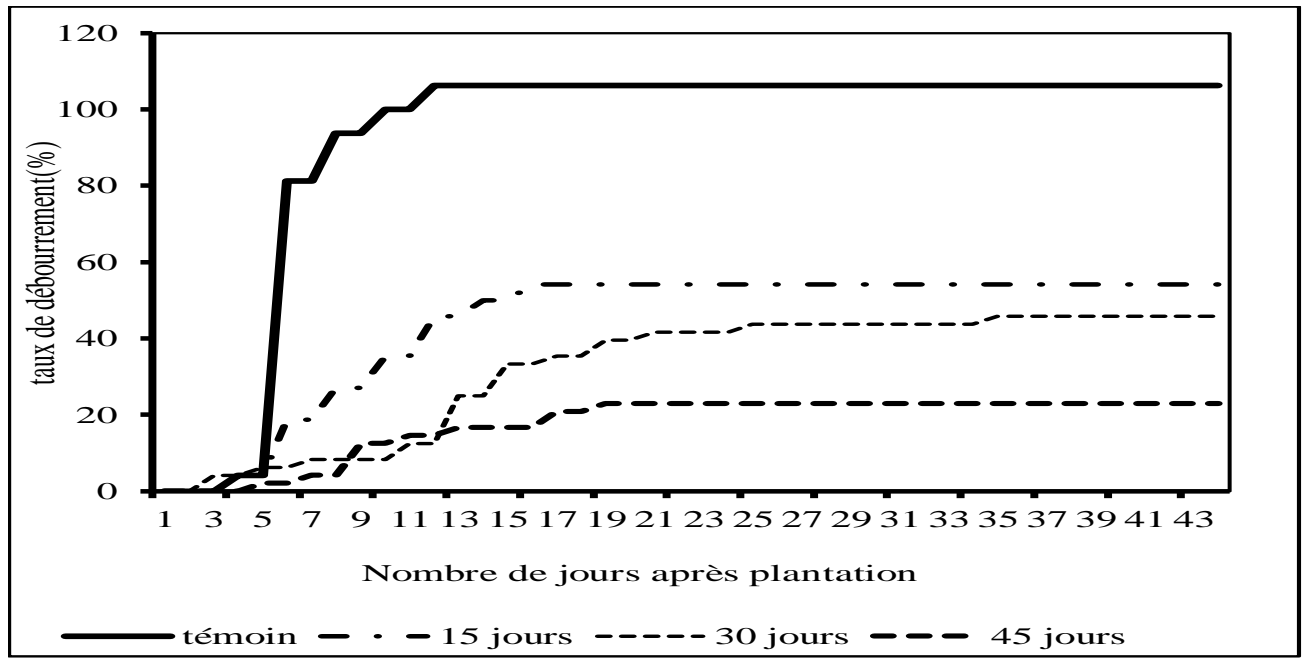

Figure 8. Cinétique de débourrement en fonction du temps de conservation 


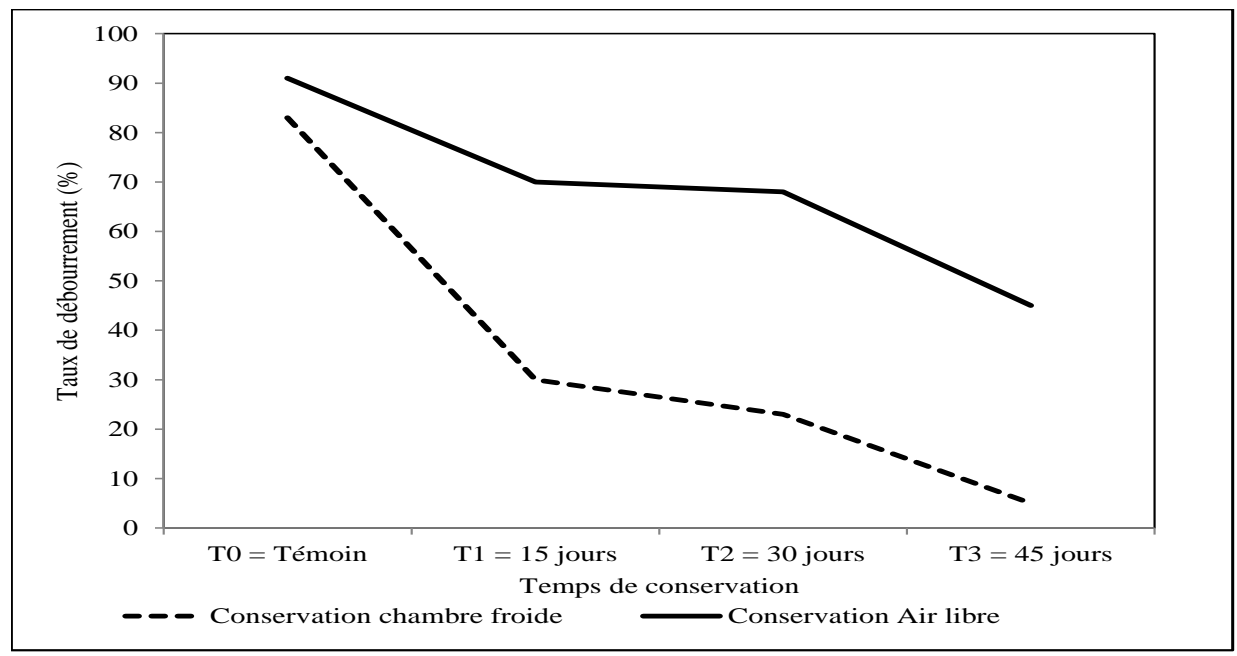

Figure 9. Evolution du débourrement des boutures en fonction du mode et du temps de conservation

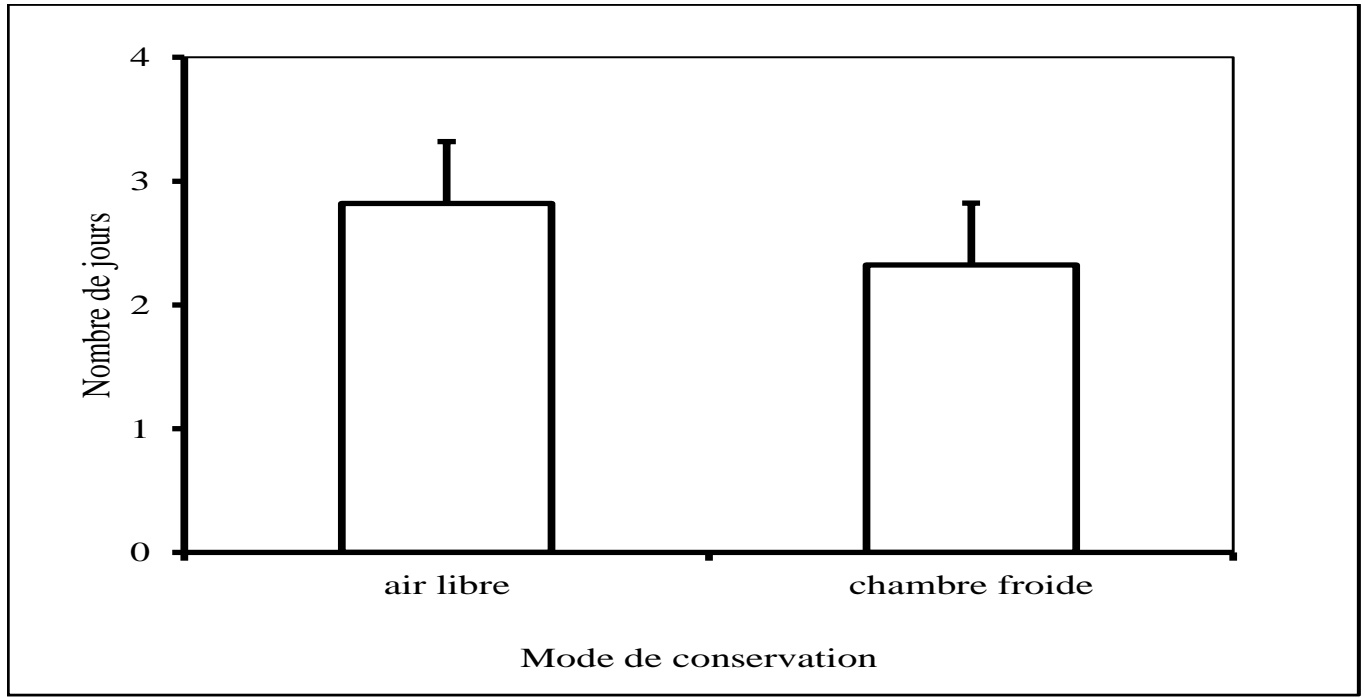

Figure 10. Influence du mode de conservation sur la durée de reprise des boutures

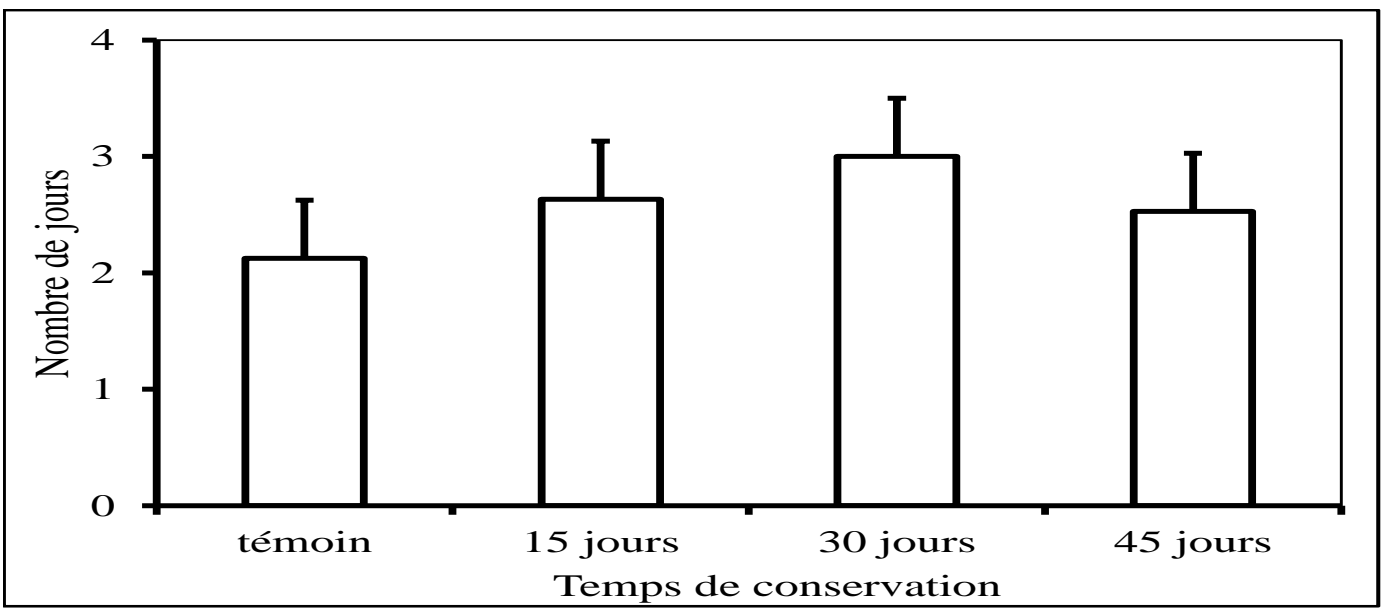

Figure 11. Effet du temps de conservation sur la durée de reprise des boutures. 


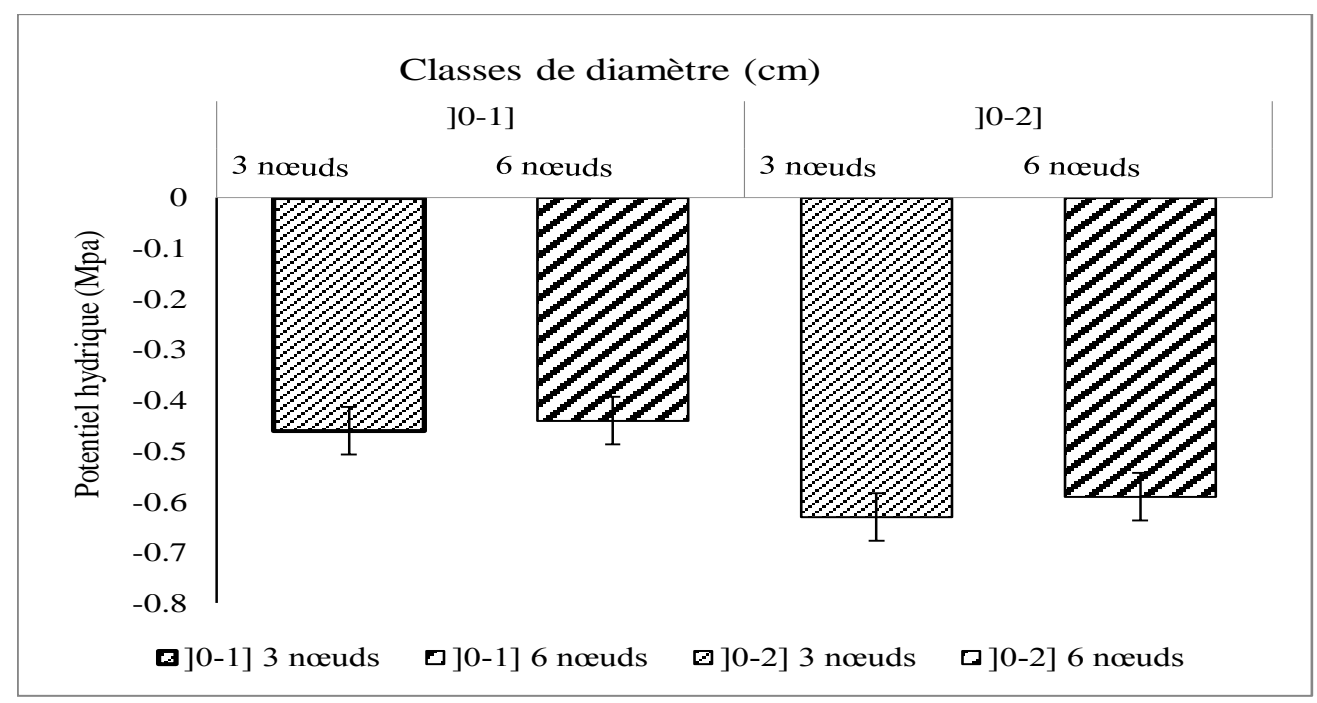

Figure 12. Potentiel hydrique en fonction des classes de diamètre et des nombres de nœuds

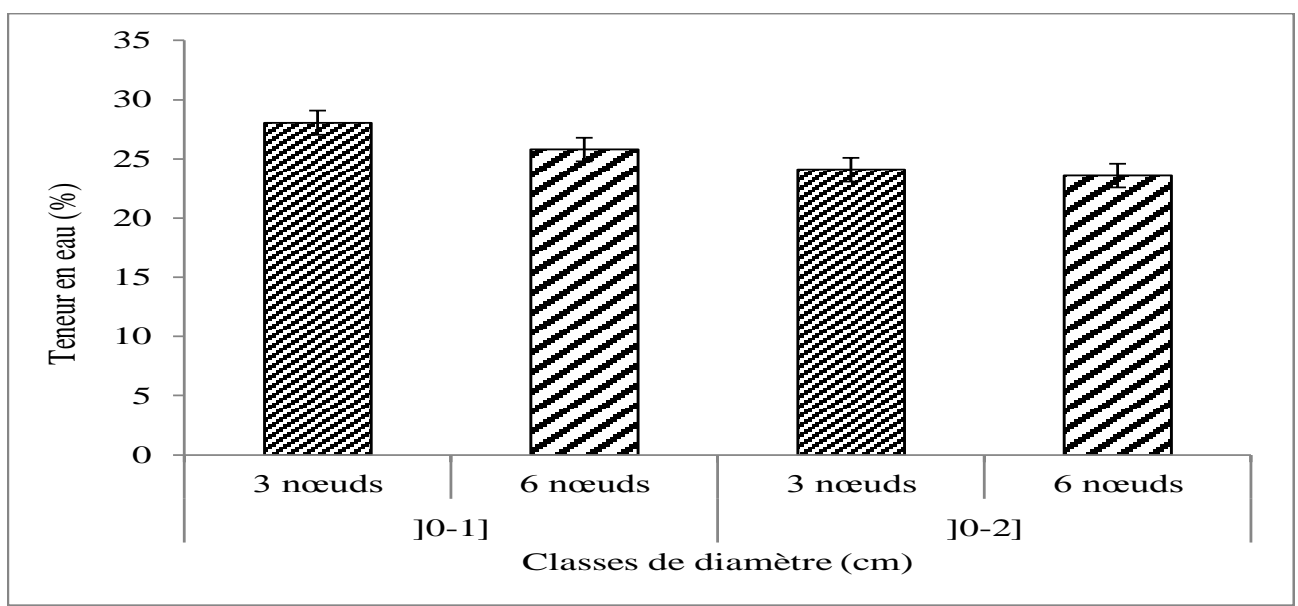

Figure 13. Teneur en fonction des classes de diamètre et des nombres de nœuds

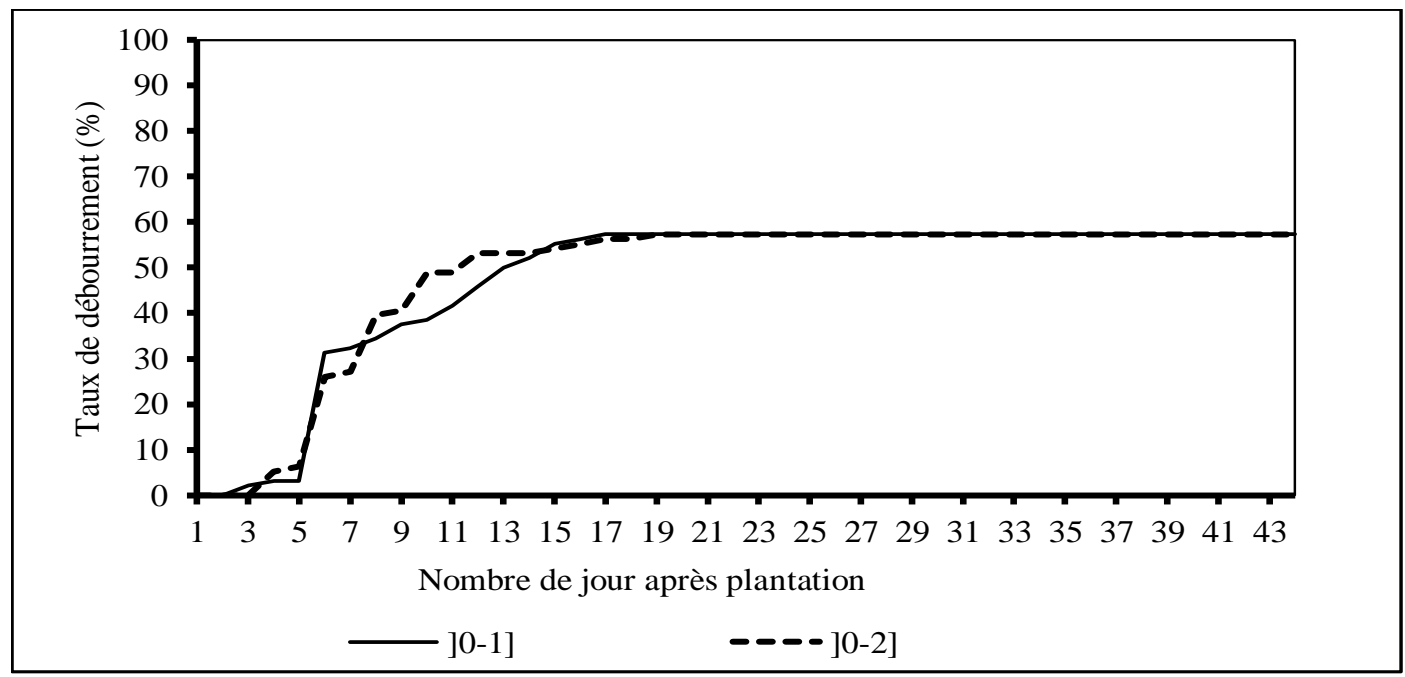

Figure 14. Cinétique du taux de débourrement des boutures en fonction des classes de diamètre. 


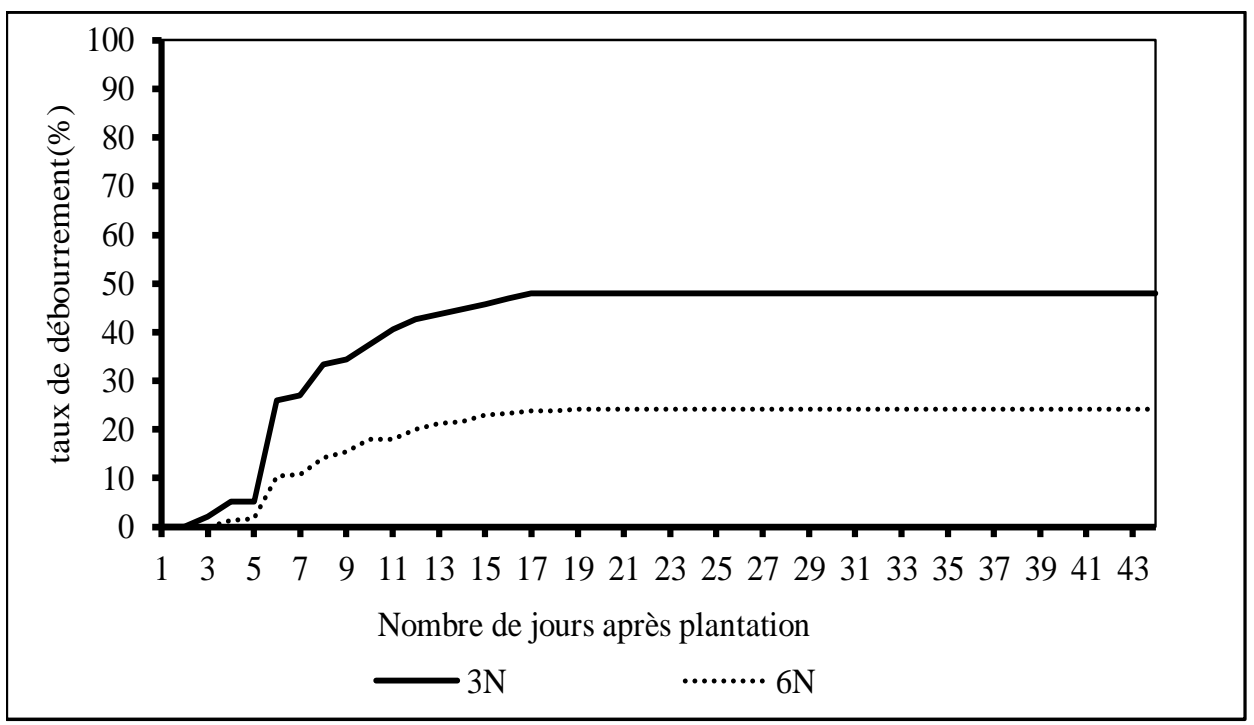

Figure 15. Cinétique de débourrement des boutures de en fonction du nombre de nœuds.

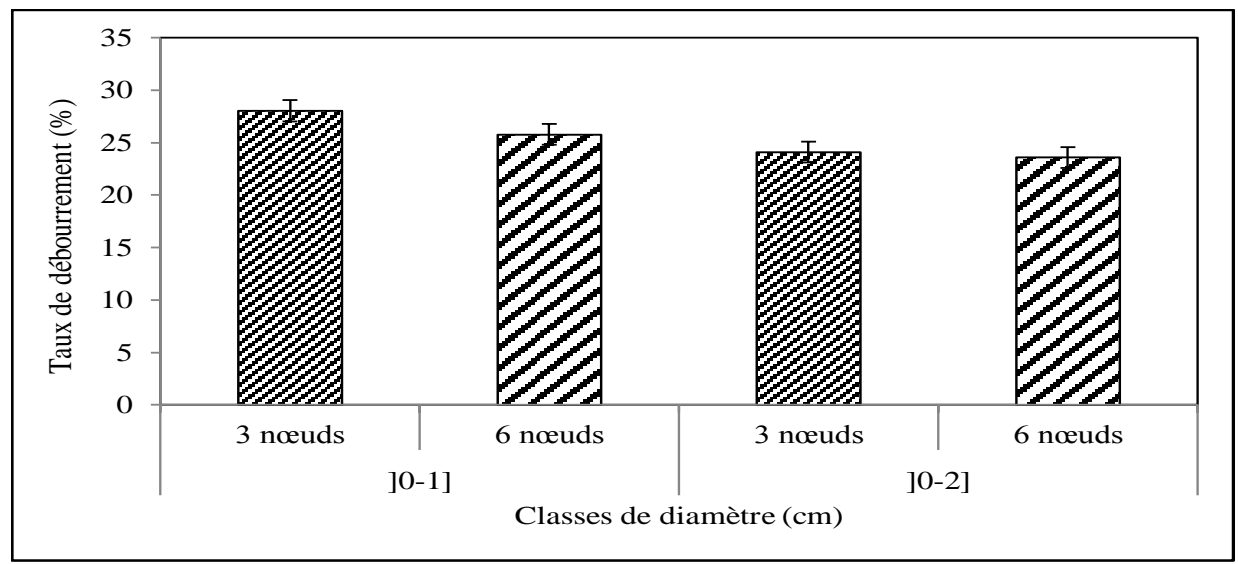

Figure 16. Taux de débourrement en fonction des classes de diamètre et du nombre de nœuds.

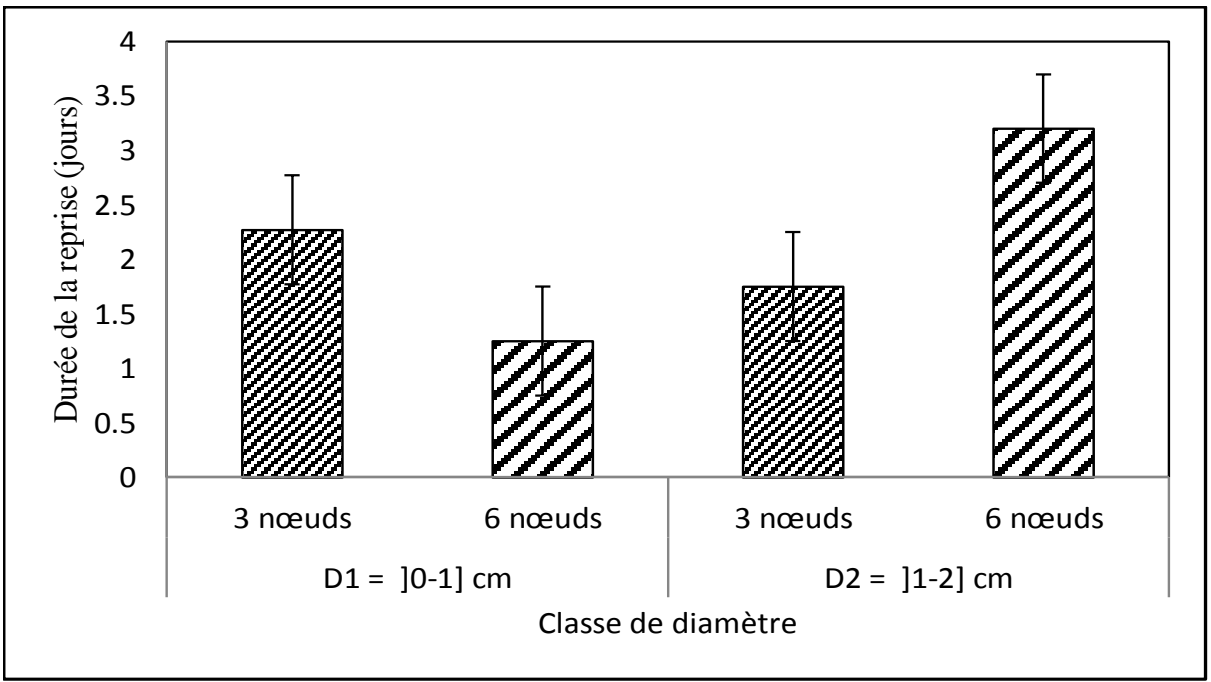

Figure 17. Durée de la reprise en fonction des classes de diamètre et du nombre de nœuds 


\section{DISCUSSION}

\section{L'état hydrique des boutures}

Etat hydrique des boutures en fonction du mode et du temps de conservation

Il ressort de cette étude que les boutures de Jatropha curcas L. se comportent différemment suivant le mode et le temps de conservation auxquels elles sont soumises. Des variations de leur statut hydrique ont été observées selon que les boutures ont été conservées en chambre froide ou à l'air libre. On a noté une augmentation de la teneur en eau pour les boutures conservées en chambre froide $(86,93 \%)$, alors que celles conservées à l'air libre voient leur teneur en eau diminuer au fur et à mesure que le stress hydrique provoqué par le temps de conservation devient plus long $(67,31 \%)$. Cet effet est réversible lors de l'évaluation du statut hydrique des boutures avec le potentiel hydrique, qui montre une augmentation pour les boutures conservées en chambre froide $(-0,33 \%)$, et une diminution pour celles conservées à l'air libre $(-0,69 \%)$.

Les mesures hydriques effectuées sur les boutures ont permis d'évaluer leur capacité à se gorger d'eau ou à en perdre dans leurs tissus par rapport à différents niveaux de stress. Les boutures calibrées ont répondu différemment face aux modes de conservation. En effet l'évaluation du niveau hydrique effectuée sur les boutures a montré que les boutures comprises entre]0-1] $(\mathrm{cm})$ ont enregistré la meilleure capacité de rétention d'eau que les boutures comprises entre]1-2] (cm).

\section{Le débourrement des boutures \\ Le mode de conservation sur le taux de débourrement}

Les boutures conservées en chambre froide et à l'air libre ont subi toutes les deux une régression de leur taux de débourrement au fur du temps (témoin ; 15 jours ; 30 jours ; 45 jours). Malgré cette diminution, les boutures conservées à l'air libre ont enregistré un taux de débourrement beaucoup plus important $(92,5 \%, 70,83 \%, 67,5 \%$ et $42,08 \%$ respectivement), alors que celles conservées en chambre froide ont enregistré des taux respectifs de $85 \%, 32,5 \%, 25 \%$ et $0 \%$ pour les mêmes temps de conservation. Ces résultats ont montré que l'effet de la conservation a une influence sur le taux de débourrement des boutures. Des études faites par Dussert et al. (2006) sur la germination des semences de Coffea arabica conservées avant semis ont monté que lorsque les semences sont conservées pendant 1 an à $5{ }^{\circ} \mathrm{C}$, leur viabilité apparait très altérée en comparaison des semences conservées à $20^{\circ} \mathrm{C}$.

La dominance du taux de débourrement des boutures conservées à l'air libre sur celles conservées en chambre froide serait due au fait que Jatropha curcas L. est une espèce tropicale tolérante à la sécheresse. Les travaux de Diop et al (2012) ont montré que les segments de rameau proches de la tige débourrent mieux (80\%) que les segments apicaux (50\%). Avec le colatier, Cola acuminata, des taux supérieurs à $80 \%$ ont été obtenus par une multiplication végétative par bouturage. (Paluku et al., 2018) Selon Barbier (2012), Jatropha curcas L. est capable de résister à une saison sèche plus ou moins prononcée avec des températures moyennes variant de $20{ }^{\circ} \mathrm{C}$ à $32{ }^{\circ} \mathrm{C}$. Son tronc à caudex qui constitue un réservoir d'eau, est capable de résister à des périodes de sécheresse prolongées (Formad environement, 2013). Cette aptitude confirme sa particularité d'espèce succulente qui lui permet de conserver sa vigueur génétique même si cette dernière régresse au fur et à mesure que les conditions de stress deviennent de plus en plus accrues. Des recherches effectuées par Jaoudi (2010) sur l'étude de la germination des graines de Acacia tortilis sous différentes contraintes abiotiques, ont montré que l'influence du stress hydrique sous l'effet de différents traitements osmotique $(0,-2,-4,-6,-8$ bars) sur le taux de germination des graines, ont donné une variation involutive de $(88 \%$, $53 \%, 38 \%, 22 \%$, et $0 \%$ ). Ces résultats confirment les travaux de Samb (2015) sur le Tamarindus indica, de Djibril et al. (2005) sur 
Phoenix dactylifera L., et de Ferchichi et al. (2004) sur Cenchrus ciliaris. Contrairement à cette capacité d'adaptation en milieu aride, les boutures de Jatropha curcas L. sont très sensibles au froid. Son air de répartition l'affirme car d'après Rijssenbeek, cité par Pirot et Hamel (2012) son aire de culture se trouve entre la latitude $30^{\circ} \mathrm{N}$ et $35^{\circ} \mathrm{S}$. Cette intolérance au froid s'est vérifiée sur nos résultats qui montrent une variation régressive du taux de débourrement au fur et à mesure que les boutures sont conservées en chambre froide. Les perturbations du métabolisme cellulaire associées à la déshydratation de la cellule et aux dommages mécaniques aboutissent alors à la déstructuration et à la mort des cellules. Les dégâts causés par les « stress » thermiques dépendent aussi du mode et de la durée de conservation, car d'après nos résultats, la variation du taux de débourrement des boutures a subi une évolution régressive et finit par s'annuler après 45 jours de conservation $(85 \%, 32,5 \%$, $25 \%$ et $0 \%$ ). Ces résultats indiquent que les boutures du Jatropha curcas L. ne sont pas très tolérantes au froid, contrairement aux graines d'après les travaux Samba et al. (2006), qui avait utilisé des graines stockées dans une chambre froide à $4{ }^{\circ} \mathrm{C}$ pendant 90 jours avant semis. Le pourcentage de germination de ces graines de Jatropha curcas L. dans des conditions de serre avait atteint un taux de germination cumulatif de $86 \%$ en 15 jours après semis. Cette divergence est due au fait qu'en dépit des graines qui sont de type orthodoxe, les boutures du Jatropha curcas L. sont récalcitrantes.

\section{La cinétique du débourrement}

L'évolution du débourrement des boutures du Jatrophas curcas L. a montré des effets différents face aux différents traitements. En effet, les boutures conservées en chambre froide ont montré un délai de débourrement beaucoup plus long (5 jours) que celles conservées à l'air libre (3 jours). Des études effectuées par Sanogo et al. (2013) sur le Carapa procera ont montré que la diminution du taux de germination après dessiccation des graines est accompagnée d'une augmentation de leur durée de germination. Les travaux menés par Diatta et al. (2007) sur le Maerua crassifolia ont montré que la durée des reprises est d'environ 3 semaines et elle est plus élevée chez les boutures proximales, particulièrement au cours du mois de juin et juillet.

\section{Le nombre de noeuds sur le débourrement des boutures}

Le nombre de nœuds a une influence très significative sur le taux de débourrement des boutures $(p=0,0000)$. Les boutures à 3 nœuds ont eu un taux de débourrement plus élevé (48\%) que celles à 6 nœuds (24\%). Ces résultats sont plutôt proches de celui de $\mathrm{B}$ Laribi et al. (2012) qui ont trouvé lors de la multiplication par bouturage d'un écotype tunisien d'Eglantier (Rosa canina L.) un taux de survie de plus de $(80 \%)$ pour les boutures semis ligneuses, (62\%) pour les boutures ligneuses. En effet, la majorité des boutures à 6 nœuds ont été prélevées à la base des rameaux constitués de cellules vieilles où l'on constate une forte concentration de nœuds. Contrairement à ces dernières, celles à 3 nœuds sont prélevées au niveau de l'apex constituées de cellules jeunes. Donc, cette dominance pourrait également être liée à leur niveau de prélèvement. Malgré cette différence, les deux catégories de boutures ont eu des courbes de débourrement cumulatif de même allure.

\section{Conclusion}

Les résultats obtenus au cours de cette étude ont montré que le statut hydrique varie en fonction du mode mais aussi du temps de conservation. Sur le plan physiologique, l'état hydrique des boutures de Jatropha curcas L. montre pour les deux modes de conservation une différence hautement significative. Par ailleurs, les résultats obtenus sur le taux de débourrement ont varié en fonction du mode de conservation. Les boutures ont enregistré pour les deux modes de conservation une variation involutive qui diminue lorsque le 
temps de conservation augmente. Mais malgré cette décroissance, les boutures conservées à l'air libre ont enregistré un taux de débourrement plus élevé que celles conservées en chambre froide qui connaissent un seuil de conservation après 45 jours. Le calibrage des boutures du Jatropha curcas L. constitue aussi un autre facteur limitant car le taux de débourrement est plus important chez les boutures à 3 nœuds (48\%) au moment où les boutures à 6 nœuds enregistrent de faible taux de débourrement (23\%), et cela par rapport à toutes les classes de diamètres confondues.

\section{CONFLIT D'INTERETS}

Aucun conflit d'intérêts n'est signalé par les auteurs.

\section{CONTRIBUTIONS DES AUTEURS}

Les travaux de terrain (collecte des boutures ainsi que l'installation de la pépinière) ont été réalisés $\mathrm{AN}, \mathrm{AT}$ avec l'appui du personnel technique de l'Inspection des Eaux et Forêts. Le protocole et la méthodologie ont été pris en charge par AT, SANS. L'analyse des résultats ainsi que la rédaction du manuscrit ont été réalisés par $\mathrm{AT}, \mathrm{COS}$ et AN.

\section{REMERCIEMENTS}

Nous remercions l'Inspection des Eaux et Forêts de Thiès (Sénégal) ainsi que le personnel technique et scientifique du CERAAS (Centre d'Etude Régional pour l'Alimentation à l'Adaptation à la Sécheresse) et le CNRF (Centre National de Recherches Forestières de l'ISRA (Institut Sénégalais de Recherches Agricoles) d'avoir bien voulu accueillir ce travail de recherche.

\section{REFERENCES}

Achten WMJ. 2008. Jatropha bio-diesel production. Bioenergy, 32 : 1063-1084.

Arnaud Wendpouire AZ, Bationo BA, Some AN, Bellefontaine R. 2014. Architecture racinaire et aptitude au drageonnage de trois espèces forestières. Int. J. Biol.
Chem. Sci., 8(3): 903-915. DOI: http://dx.doi.org/10.4314/ijbcs.v8i3.7

Barbier. 2012. Intérêt de mettre en place une filière courte basée sur la culture du Jaropha (Jatropha curcas) dans la communauté rurale de Dialacoto Sénégal. Document de synthèse de recherche :

http://orbi.ulg.ac;be/bitstream,

2268/13282/1/20120703\% 20document $\% \quad 20$ Synthèse $\% \mathrm{C} 3 \% \quad \mathrm{~A} 8 \mathrm{x} \% \quad 20$ Jatropha $\% 20$ vf.pdf, (24/04/2013).

Bayoulou S. 2013. Effet de la culture du jatropha sur les caractéristiques chimiques des sols dans la zone Ouest du Burkina Faso: Cas des sols de Tin et de Torokoro. 43 p. Mémoire de Master en Science du Sol. Université Polytechnique de Bobo-Dioulasso. Institut du Développement Rural.

Bazongo P, Traoré K, Traoré O, Bilgo A, Yélémou B, Sanon KB, Hien V, Nacro BH. 2015. Caractérisation des systèmes de production de Jatropha dans les exploitations agricoles de la zone Ouest du Burkina Faso. Int. J. Biol. Chem. Sci., 9(5): $2432-2445 . \quad$ DOI: http://dx.doi.org/10.4314/ijbcs.v9i5.15

CIRAD, MNHN, UICN. 2012. Montpellier, France. 1ère Ed. $241 \mathrm{p}$

Formad-environnement. 2013. Jatropha. https://www.formad-

environnement.org/formad-jatropha-

2013.pdf 21 pages; Consulté le 09/09/2018

SIE-Sénégal. 2010 Rapport système d'information énergétique du Sénégal www.compagnie3e.com/images/stories/d ocumentation/.../Rapport_SIE_Senegal.p df 61 pages; Consulté le 10/07/2018

Dereuddre J, Gazeau C. 1992. Les végétaux et les très basses températures. Les Végétaux et le Froid. Ed Herman ; 107175.

Diatta S, Houmey VK, Akpo LE, KaboréZoungrana CY, Banoin M. 2007 Possibilités de bouturage chez Maerua crassifolia Forssk., Capparaceae, un 
ligneux fourrager Sahélien. Afrique SCIENCE, 03(2) : 271 - 283.

Diop B, Samba ANS, Akpo LE. 2012. Caractéristiques morphologiques et croissance de jeunes plants de Jatropha curcas L. Int. J. Biol. Chem. Sci., 6(2): 677-691.

DOI: http://dx.doi.org/10.4314/ijbcs.v6i2.12

Dusserts S, Davey MW, Laffrargue A. 2006. Oxidative stress, phospholipid loss and lipid hydrolysis during drying and storage of intermediate seeds. Physiologia Plantarum, 127(2) : 192-204.

DOI : https://doi.org/10.1111/j.1399-

3054.2006.00666.x.

Ecomet. 2014. Evaluation du risque environnemental lié à l'utilisation des pesticides dans les zones https://www.programmepesticides.fr/Pag es-projets/APR-20062/ECOMET.

Henning RK. 2000. The Jatropha Booklet: A guide to the Jatropha system and its dissemination in Africa. Bagani GbR, Weissensberg. $37 \mathrm{p}$.

Jaouadi W, Hamrouni L, Souayeh N. 2010. Etude de la germination des graines d'Acacia tortilis sous différentes contraintes abiotiques. Biotechnologie, Agronomie, Société et Environnement, 14(4) : 643-652.

Kẹpczyński J. 1986. Inhibition of Amaranthus caudatus seed germination by polyethylene glycol-6000 and abscisic acid and its reversal by ethephon or 1aminocyclopropane-1-carboxylic acid. Physiologia Plantarum, 67(4): 588-591. DOI : https://doi.org/10.1111/j.13993054.1986.tb05060x.

Laribi B, Touil R, Kouki K, Bettaieb T. 2013. Multiplication par bouturage d'un écotype Tunisien d'Eglantier (Rosa canina L.). Nature \& Technology, BSciences Agronomiques et Biologiques, 09: 55-59.

Mazur P. 1984. Freezing of living cells mechanisms and implications. American journal of Physiology-cell Physiology, 247(3) C125-C142. Https// doi.org/10.1152/ajpccell. 1984.247.3. C125.

Meryman HT, Williams RJ, Douglas MSJ. 1977. Freezing injury from "solution effects" and its prevention by natural or artificial cryoprotection. Cryobiology, 14(3): 287-302. DOI: https://doi.org/10.1016/00112240(77)90177-8.

Mnif L, Chaieb M, Ferchichi A. 2004. Comportement germinatif de différentes provenances de Cenchrus ciliaris L. collectées de la zone aride Tunisienne. Réhabilitation des pâturages et des parcours en milieux méditerranéens. Cahiers Options Méditerranéennes, 62 : 107-111.

Nguema N, Bouanga EB, Massounga YC, Boussiengui Boussiengu G. 2013. Étude comparée de trois méthodes de multiplication de Jatropha curcas L. dans les conditions climatiques du sudest du Gabon. Journal of Applied Biosciences, 65: 4989 - 4998.

Openshaw K. 2000. A review of Jatropha curcas: an oil plant of unfulfilled promise. Biomass and Bioenergy, 19(1): 1-15.

DOI: https://doi.org/10.1016/S09619534(00)00019-2.

Paluku A, Bwama M, Okungo A, Van Damme P. 2018. Bouturage de Cola acuminata (P. Beauv.) Schott \& Endl.: Influence du substrat, de la longueur et de la surface foliaire sur l'enracinement de boutures à Kisangani, RD Congo. Int. J. Biol. Chem. Sci., 12(3): 1141-1150, DOI:

https://dx.doi.org/10.4314/ijbcs.v12i3.6.

Pirot R., Hamel O. 2012. Les réalités du Jatropha curcas confrontées aux opportunités des mécanismes financiers liés au carbone. (Fantasme, aubaine ou réelle opportunité ?). Crédits carbone pour l'Agriculture, la Sylviculture, la Conservation et l'Action contre la 
Déforestation (CASCADE), Rapport Cirad. France. 32 p.

Rijssenbeek W, Galema T. 2010. The jatropha handbook from cultivation to application. Eindhoven the Ntherlands. Fact. Foundation 29-37.

Samb CO. 2015. Etude de la levée de dormance et de la germination de cinq provenances de Tamarindus indica L. en conditions de stress hydrique au Sénégal. Mémoire de fin d'étude pour l'obtention du diplôme de Master en Foresterie et Environnement pour une Gestion Durable des Ressources Naturelles, ENSA, Université de Thiès, 26p.

Samba ANS, Diallo B, Diop M, Diatta M, Sarr S, Diouf M. 2006. Seed germination and propagation methods. Institut Sénégalais de Recherches Agricoles (ISRA) - Laboratoire National des Recherches sur les Productions Végétales (LNRPV), Route des Hydrocarbures, Bel-Air, BP 3120 Dakar. $17 \mathrm{p}$.

Samba ANS, Diallo B, Sané D, Diop T. 2013. Effet du chlorure de sodium sur la germination de graines de Ricinus communis L. Int. J. Biol. Chem. Sci.,
7(4):

1534-1544.

DOI:

http://dx.doi.org/10.4314/ijbcs.v7i4.10

Sané D, Ould Kneyta M, Diouf D, Diouf D, Badiane FA, Sagna M, Borgel A. 2005. Growth and development of date palm (Phonix dactylifera L.) seedlings under drought and salinity stresses. African Journal of Biotechnology, 4(9): 968-972.

Sanogo S, Sacandé M, Van Damme P, Diaye IN. 2013. Caractérisation, germination et conservation des graines de Carapa procera DC. (Meliaceae), une espèce utile en santé humaine et animale. Biotechnologie, Agronomie, Société et Environnement, 17(2): 321331.

Uemura M, Steponkus PL. 1999. Cold acclimation in plants: relationship between the lipid composition and the cryostability of the plasma membrane. Journal of Plant Research, 112(2): $\quad 245-254 . \quad$ DOI: https://doi.org/10.1007/PL00013882.

Willan RL. 1992. Guide de manipulation des semences forestières dans le cas particulier des régions tropicales. Rome : FAO. (Etude FAO : Forêts, Volume 20). 